\section{Pacific Northwest}

National Laboratory

Operated by Battelle for the

U.S. Department of Energy

\section{Long Wave Infrared Detection of Chemical Weapons Simulants}

\author{
M. C. Phillips \\ M. S. Taubman \\ D. C. Scott \\ T. L. Myers \\ J. T. Munley \\ B. D. Cannon
}

April 2007

Prepared for the U.S. Department of Energy under Contract DE-AC05-76RL01830 


\title{
Long Wave Infrared Detection of Chemical Weapons Simulants
}

\author{
M. C. Phillips \\ M. S. Taubman \\ D. C. Scott \\ T. L. Myers \\ J. T. Munley \\ B. D. Cannon
}

April 2007

Prepared for the U.S. Department of Energy

Under Contract DE-AC05-76RL01830

Pacific Northwest National Laboratory

Richland, Washington 99352 


\section{Contents}

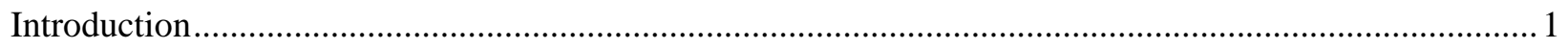

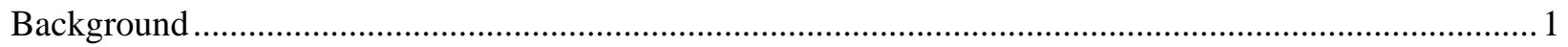

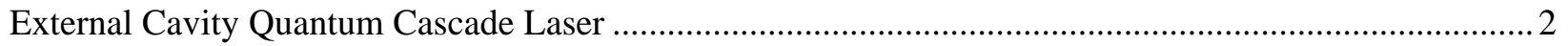

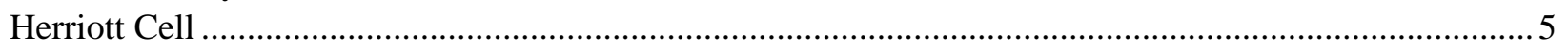

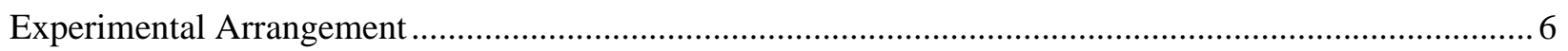

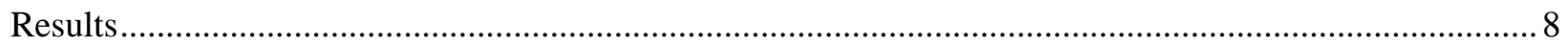

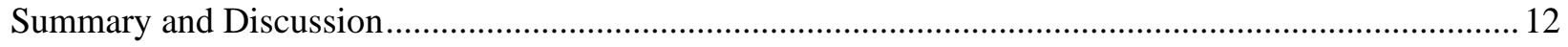

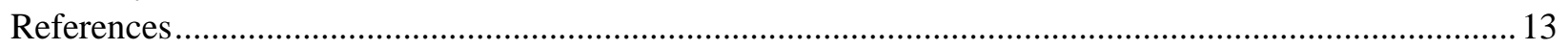

\section{Figures}

1. Schematic of Littman-Metcalf External Cavity Configuration...................................................... 3

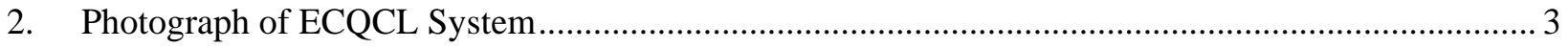

3. FTIR Spectra of the ECQCL Output for Eight Different Tuning Mirror Positions............................. 4

4. Calibration of ECQCL Output Frequency to Stepper Motor Position............................................... 4

5. Measured Average Output Power and SMSR over the ECQCL Scan............................................. 5

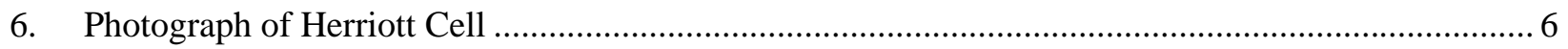

7. Photograph of Herriott Cell C-3 Mode with the Green Alignment Laser .......................................... 6

8. Experimental Arrangement Used for Freon-125 Spectral Measurements ........................................... 7

9. Measured Absorption Spectrum of 10 ppm Freon-125 in Fast Scan Mode......................................... 9

10. Fast Scan Absorption Spectrum of 10 ppm Freon-125................................................................... 10

11. High Resolution Absorption Spectrum of 10 ppm Freon-125 ........................................................ 10

12. Individual Scans Used to Calculate Absorption Spectrum ............................................................ 11

13. Absorption Spectrum of Room Air in Herriott Cell ...................................................................... 12 



\section{Acronyms}

\begin{tabular}{|c|c|}
\hline AR-coated & anti-reflection coated \\
\hline CBW & Chemical/Biological Weapons \\
\hline DFB & distributed grating feedback \\
\hline ECQCL & external cavity quantum cascade laser \\
\hline FP & Fabry-Perot \\
\hline FTIR & Fourier-transform infrared [spectrometer] \\
\hline $\mathrm{HeNe}$ & helium neon [laser] \\
\hline HR-coated & high-reflectance coated \\
\hline LCP & lifecycle plan \\
\hline NWIR & Northwest Infrared \\
\hline $\mathrm{NH}_{3}$ & ammonia \\
\hline $\mathrm{N}_{2} \mathrm{O}$ & nitrous oxide \\
\hline $\mathrm{O}_{3}$ & ozone \\
\hline $\begin{array}{l}\text { PNNL } \\
\text { ppbv }\end{array}$ & $\begin{array}{l}\text { Pacific Northwest National Laboratory } \\
\text { parts-per-billion by volume }\end{array}$ \\
\hline QCL & quantum cascade laser \\
\hline SMSR & side mode suppression ratio \\
\hline SNR & signal-to-noise ratio \\
\hline
\end{tabular}




\section{Introduction}

The purpose of Task 3.b under PL02-OP211I-PD07 (CBW simulant detection) was to demonstrate the applicability of the sensor work developed under this project for chemical and biological weapons detection. To this end, the specific goal was to demonstrate the feasibility of detection of chemical agents via that of simulants (Freons) with similar spectroscopic features. This has been achieved using Freon125 as a simulant, a tunable external cavity quantum cascade laser (ECQCL), and a Herriott cell-based sensor developed at Pacific Northwest National Laboratory (PNNL) specifically for this task. The experimentally obtained spectrum of this simulant matches that found in the Northwest Infrared (NWIR) spectral library extremely well, demonstrating the ability of this technique to detect the exact shape of this feature, which in turn indicates the ability to recognize the simulant even in the presence of significant interference. It has also been demonstrated that the detected features of a typical interferent, namely water, are so different in shape and width to the simulant, that they are easily recognized and separated from such a measurement.

Judging from the signal-to-noise ratio (SNR) of the experimental data obtained, the noise equivalent absorption sensitivity is estimated to be $0.5 \times 10^{-7}$ to $1 \times 10^{-6} \mathrm{~cm}^{-1}$. For the particular feature of the simulant examined in this work, this corresponds to a relative concentration of 50 to 25 parts-per-billion by volume (ppbv). The corresponding relative concentrations of other chemical targets would differ depending on the particular transition strengths, and would thus have to be scaled accordingly.

\section{Background}

Quantum cascade lasers (QCLs) are a technology that has allowed relatively easy access to the molecular fingerprint region of the infrared spectrum. They are robust monopolar semiconductor laser devices that can be fabricated to operate at specific wavelengths virtually anywhere in the 4- to 20-micron range. Until recently, all of the work performed under the sensors task of PL02-OP211I-PD07 was done using distributed grating feedback (DFB) QCLs, allowing narrow line width operation and access to narrow features of simple molecules such as $\mathrm{N}_{2} \mathrm{O}$ and $\mathrm{NH}_{3}$. This work has been covered extensively in our annual reports for the Long Wave Infrared Cavity Enhanced Sensors task (Taubman et al. 2003a; Taubman et al. 2003b; Taubman et al. 2004; Taubman et al. 2005). For the current CBW task, however, the target features are far too broad to allow the use of such narrow-band lasers. This is where the broad tuning capabilities of an ECQCL are required. ECQCLs have been under development at PNNL for a number of years, and their design has been covered in the Laser Development annual reports (Phillips et al. 2005). The laser used is this case is similar to those designs and allows a scan range of $65 \mathrm{~cm}^{-1}$, which is more than adequate to scan the absorption features of the chosen Freon-125 simulant. (This is in contrast to the typical $2 \mathrm{~cm}^{-1}$ tuning of a DFB QCL.)

The Herriott cell used in the sensor was also designed and constructed at PNNL, and is similar to that used in Task 3a of the project. The basic construction of a Herriott cell is to place two curved mirrors a

fixed distance apart using stable mounts or separators. Light is then passed into the cell through a hole in one mirror, which is then bounced multiple times between the mirrors, to finally exit at the same hole through which it entered, but at a different angle. There are several different configurations for Herriott cells depending on the relationship between the curvature of the mirrors and their separation, resulting in 
different bounce patterns or geometric modes. The chosen mode of the Herriott cells currently in use at PNNL is the C-3 mode, as this affords stability against lateral movement of the mirrors with respect to one another.

\section{External Cavity Quantum Cascade Laser}

External cavity semiconductor lasers differ from simple cavity laser devices in that the front facet of the semiconductor material is usually anti-reflection coated (AR-coated) to prevent reflection from this surface. This allows the optical cavity responsible for the lasing feedback action to be formed between the back surface of the semiconductor device, usually a high-reflectance coated (HR-coated), and an external reflecting optic such as a mirror. This has many advantages, including reduced operating line width simply due to the vastly increased cavity length as compared to that normally formed within the bounds of the semiconductor device itself. A more powerful advantage is the ability to use specialized optics for the external element, such as a grating. This allows only specific optical wavelengths to reflect at certain angles, thus providing much enhanced frequency selection. Moreover, turning or tilting the grating allows the lasing wavelength to be tuned by changing the angle of reflection. This alters the selected wavelength to be fed back into the optical cavity for amplification by the laser medium, thus allowing the lasing wavelength to be tuned. The breadth of this tuning effect is in theory limited only by the width of the gain bandwidth of the laser medium, although coating bandwidths and quality of the involved optics also play an important part.

There are several different configurations for external cavity lasers. The ECQCL developed at PNNL and used for the CBW task was based on the Littman-Metcalf configuration (Littman and Metcalf 1978), which uses both a grating and a mirror to achieve tuning without adversely steering the output beam. In this arrangement, shown in Figure 1, it is not the grating that is tilted but the mirror. Since light exiting the QCL medium is rapidly diverging, a focusing lens adjacent to the QCL was necessary to collimate the beam (0.8 numerical aperture germanium aspheric, 0.5-in. diameter). The grating used had 150 grooves per $\mathrm{mm}$, and was blazed for an optimum wavelength of $6 \mu \mathrm{m}$. The first-order diffraction beam was reflected by the tuning mirror, whose angle selected the feedback wavelength of the laser. The mirror was held in a standard mirror mount with its angle controlled using a commercial stepper motor actuator (Thorlabs EAS503) and controller (Thorlabs BSC100). The direct reflection from the grating or "zerothorder” diffraction beam was used as the laser output, and did not steer as the mirror was turned.

The QCL, obtained from Maxion Technologies (FQ8-M446J), is labeled in Figure 1 as "FP QCL". The FP stands for Fabry-Perot and indicates that there is no DFB grating deposited onto the laser chip, its absence allowing full access to the gain bandwidth of the medium, and relinquishing frequency selection and control to the external grating-mirror combination. The device was provided to us with an HRcoating on the back facet and an AR-coating on the front facet of $1 \%$ estimated reflectivity. The QCL was mounted on a copper c-mount, which was then mounted to a thermoelectric cooler to provide thermal stabilization and heat extraction from the laser. The QCL was held at $15^{\circ} \mathrm{C}$ for the experiments reported here. 


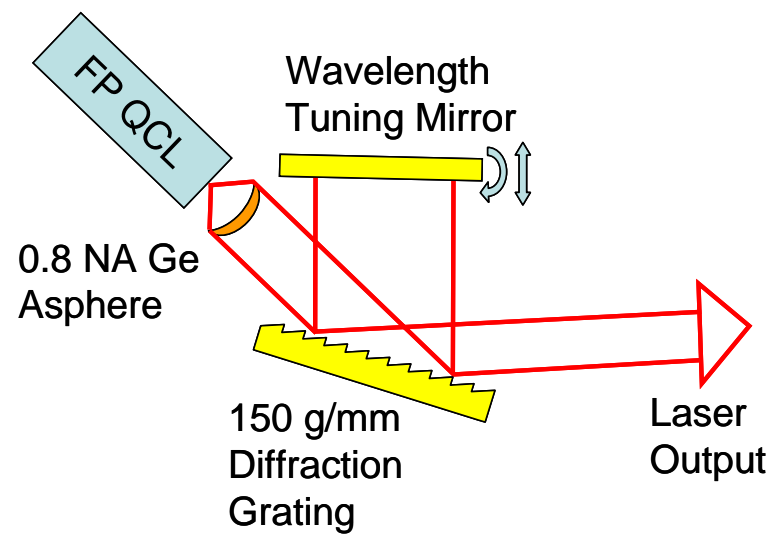

Figure 1. Schematic of Littman-Metcalf External Cavity Configuration

The length of the external cavity formed between the back facet of the QCL and the tuning mirror was $12 \mathrm{~cm}$. The ECQCL system was mounted on a 12-in.-square breadboard. A photograph of the ECQCL system is shown in Figure 2.

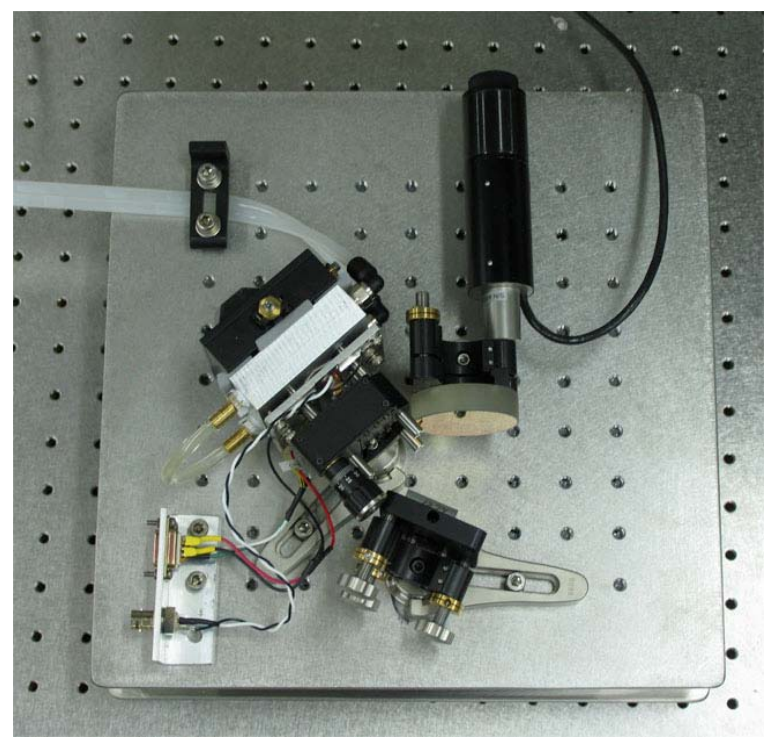

Figure 2. Photograph of ECQCL System

By adjusting the angle of the tuning mirror via the stepper motor actuator, the output frequency of the ECQCL could be tuned over the range $1120-1185 \mathrm{~cm}^{-1}$. Figure 3 shows a series of spectra measured with a Fourier-transform infrared (FTIR) spectrometer for eight different tuning mirror positions. For all tuning mirror positions within this range, the measured ECQCL line width was smaller than the FTIR resolution of $0.5 \mathrm{~cm}^{-1}$. Previous measurements have shown that the average line width of the ECQCL system is smaller than $0.2 \mathrm{~cm}^{-1}$ (Phillips et al. 2007). While the instantaneous ECQCL line width is much 
smaller than $0.2 \mathrm{~cm}^{-1}$, the average line width includes contributions from frequency chirp during the pulse and mode-hops on the external cavity modes, spaced by approximately $0.04 \mathrm{~cm}^{-1}$.

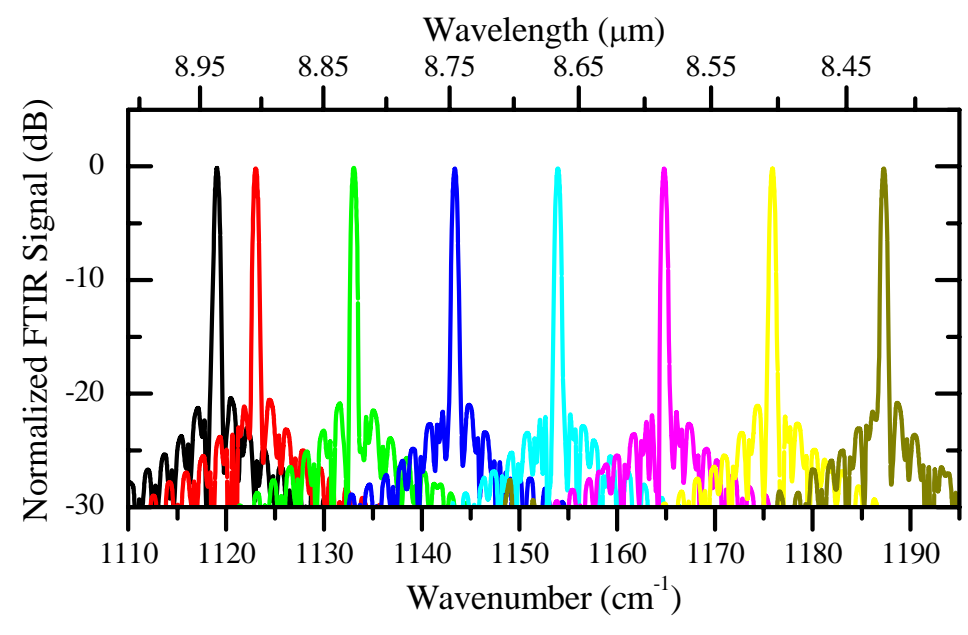

Figure 3. FTIR Spectra of the ECQCL Output for Eight Different Tuning Mirror Positions

The measured FTIR spectra were used to calibrate the ECQCL scan. Figure 4 shows the measured frequency (in wave number) from the FTIR spectra versus the stepper motor position. The curve was fitted by a third-order polynomial, which approximates the trigonometric relationship between linear motion of the stepper motor actuator and output frequency of the ECQCL. This calibration was used to express all the spectra reported here in terms of wavenumber.

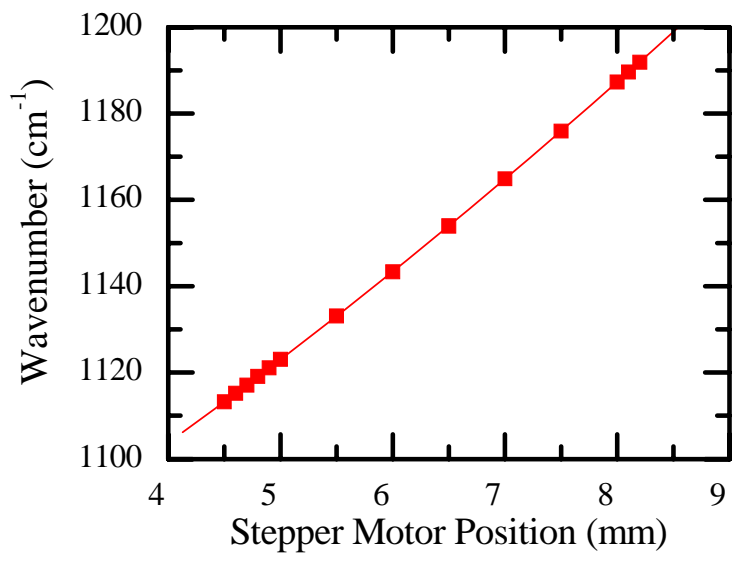

Figure 4. Calibration of ECQCL Output Frequency to Stepper Motor Position. The points show the center wavenumber of the ECQCL output measured from the FTIR spectra. The solid line is a third-order polynomial fit. 
The FTIR spectra were also used to estimate the side mode suppression ratio (SMSR) of the ECQCL system. These results are shown in Figure 5. Note that the SNR and resolution of the FTIR limited the SMSR measurement to $35 \mathrm{~dB}$. We defined the useable tuning range by the requirement that the SMSR be greater than $30 \mathrm{~dB}$, which occurred over the range 1120-1185 $\mathrm{cm}^{-1}$ (8.44-8.93 microns). The ECQCL could be operated outside this range, but with reduced performance. The average output power from the ECQCL was measured with a thermopile detector, and is also plotted in Figure 5. The power ranged from $2 \mathrm{~mW}$ to $4.5 \mathrm{~mW}$ over the scan range of the ECQCL.

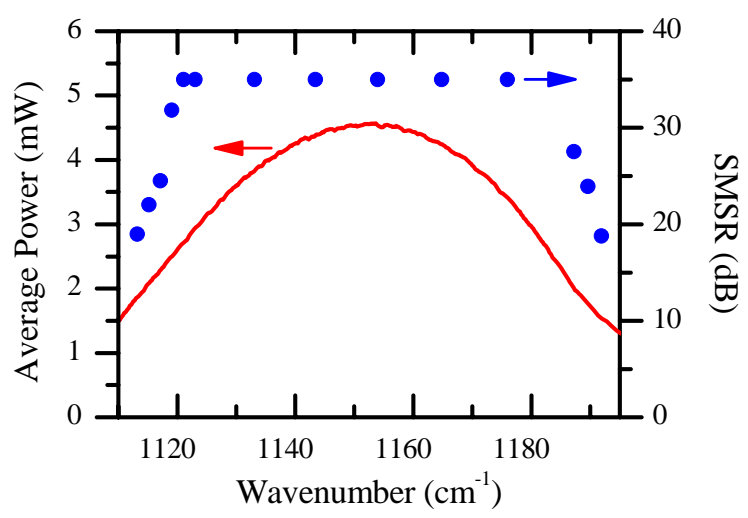

Figure 5. Measured Average Output Power (red line) and SMSR (blue points) over the ECQCL Scan. The ECQCL exhibited a SMSR $>30 \mathrm{~dB}$ over the tuning range $1120-1185 \mathrm{~cm}^{-1}$.

\section{Herriott Cell}

The Herriott cell was constructed using two 42-cm radius of curvature, 15-cm diameter mirrors mounted into machined black delrin mounts, separated by $22 \mathrm{~cm}$ using superinvar rods, and then housed inside a large plastic tube. A photo of this cell is shown in Figure 6. Analyte could be passed through the cell via the two fittings screwed into the sides of the tube, of which only one is visible in the figure. Also visible in the figure is the aperture in the front mirror and mount, which allowed entrance and exit beams to pass into and out of the Herriott cell. A wedged AR-coated zinc-selenide window, placed over this hole and in contact with an O-ring, allowed the cell to be sealed and operated at pressures down to vacuum, while allowing the optical beams to pass through freely. Figure 7 is a photograph of the Herriott cell showing the path of the green alignment laser (discussed in next section). 


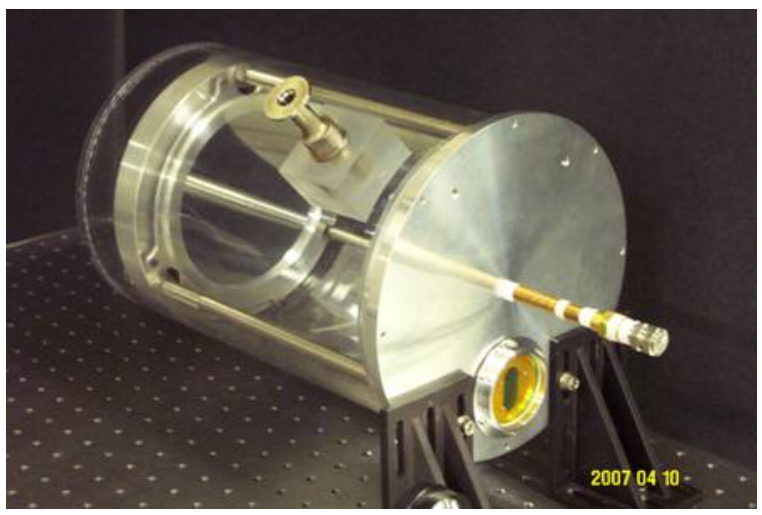

Figure 6. Photograph of Herriott Cell

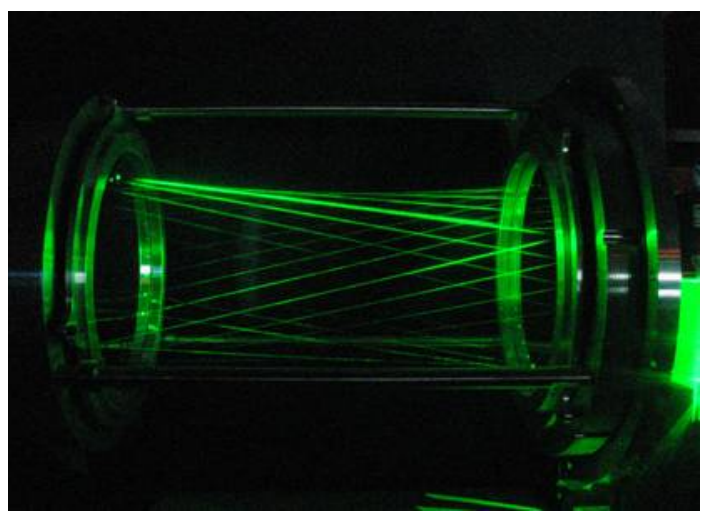

Figure 7. Photograph of Herriott Cell C-3 Mode with the Green Alignment Laser

\section{Experimental Arrangement}

Figure 8 shows the experimental arrangement used for recording spectra of Freon-125. The ECQCL was coupled into the Herriott cell using a telescope arrangement consisting of two zinc-selenide lenses (focal lengths $50 \mathrm{~mm}$ and $38 \mathrm{~mm}$ ). This telescope both reduced the beam diameter of the ECQCL, and allowed fine adjustment of the focal spot position by translating one of the lenses. This in turn allowed placement of the optimum spot size within the Herriott cell, calculated from the mirror radius of curvature and separation to be $1.43 \mathrm{~mm}$. To aid initial alignment of the Herriott cell, a green helium neon (HeNe) laser was used to obtain the desired C-3 spot pattern. The infrared beam from the ECQCL was then aligned collinearly with the HeNe. For the first experiments, the Herriott cell was configured with a mirror separation of $22.0 \pm 0.5 \mathrm{~cm}$. By counting the spots of the green laser on the mirrors, it was determined that the laser traversed the cell 58 times. Accounting for the inclination of the beam within the cell, the total path length was calculated to be $1305 \pm 30 \mathrm{~cm}$. The output from the Herriott cell was focused onto a liquid nitrogen-cooled MCT detector (Fermionics PV-12-1). To prevent saturation of the detector, a high reflectivity ( $>99 \%$ ) coated window was used to reduce the laser power.

Current was provided to the ECQCL by a custom current controller (QCL6N), developed in earlier years under the same project (Taubman et al. 2004; Taubman et al. 2005). In this case, the current controller was operated in pulsed mode; the pulses provided by a Tektronix AFG3022 function generator, and these pulses having a repetition rate of $50 \mathrm{kHz}$, a duration of 8 microseconds, and a 2 amp peak current. ${ }^{(1)}$ The signal from the detector was amplified with a custom pre-amplifier (RTIA01) also developed under the same project in earlier years, and then demodulated using a commercial lock-in amplifier (Stanford Research Systems SR830). ${ }^{(2)}$ The output of the lock-in amplifier was then passed to a

(1) The pulses were additionally shaped with a 2.5-microsecond ramp for the rising edge. Using current pulses with a slow rising edge was found to reduce the effects of lasing on QCL internal Fabry-Perot modes during the early portion of the pulse, thereby increasing the effective SMSR and tuning range of the ECQCL.

(2) A reference signal for the Lock-in amplifier was obtained from the sync output of the function generator, not shown in Figure 8. 
computer (PC), which recorded spectral information of the gaseous samples during automated laser scans, which were also controlled by the computer via the stepper motor and its controller, and the ECQCL tuning mirror.

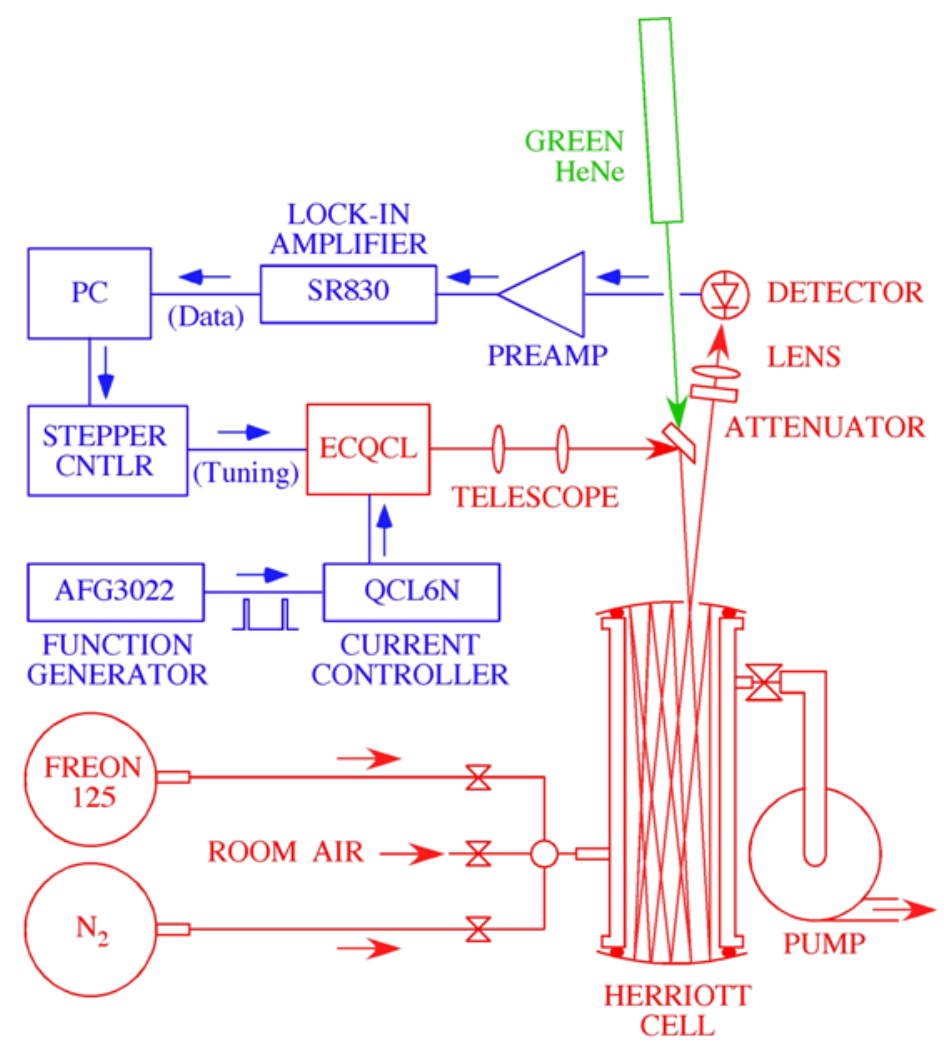

Figure 8. Experimental Arrangement Used for Freon-125 Spectral Measurements

Samples were drawn through the Herriott cell by a vacuum pump on one side of the cell, from a mixing manifold with various control valves on the other side. For gas sensing experiments, the Herriott cell was filled with a 10-ppm mixture of Freon-125 (pentafluroethane) in nitrogen, as supplied in a gas cylinder from a manufacturer. Pure nitrogen was also used for reference scans, providing an inert intracavity medium. Scans were also performed using room air, to examine the effects of atmospheric water as an interferent. To facilitate faster and more complete gas transfers, the Herriott cell was pumped out using the vacuum pump between changing gas types. All scans were performed with the Herriott cell contents at atmospheric pressure.

The time required to complete a scan depended on the scan range, the desired scan resolution, and the settling time of the lock-in amplifier used for detection (which determined the detection bandwidth). For the experiments reported here, two sets of scan parameters were used. High-resolution scans were performed by moving the stepper motor over a 3-mm distance in 2-micron increments, giving a scan resolution of $0.04 \mathrm{~cm}^{-1}$ and resulting in a spectrum with 1500 points. With a settling time of $100 \mathrm{~ms}$ at each point for electronics associated with the detection process (lock-in amplifier), the scan was completed in 180s. The extra time over the expected 150s is due to communication delays between the 
computer, stepper motor controller, and lock-in amplifier. Fast scans were performed by scanning over the same range, but with a stepper motor increment of 10 microns, resulting in a scan resolution of $0.2 \mathrm{~cm}^{-1}$ and a spectrum with 300 points. These scans were completed in 40s.

The basis of all these spectral scans involved observing the optical transmission through the Herriott cell while the ECQCL frequency was scanned. However, since the laser intensity changes as the frequency is scanned, it is difficult to measure target absorption from just one such scan with Freon-125 in the cell $\left(I_{F 125}(\bar{v})\right)$. This is especially true since the absorption feature is broad. Consequently, to obtain meaningful spectral data from Freon-125 scans, they must be compared to reference scans taken using nitrogen in the Herriott cell $\left(I_{0}(\bar{v})\right)$. This is achieved by substituting the two raw data scans into the equation for Beer's absorption law as shown below:

$$
\alpha_{10}(\bar{v})=-\frac{1}{L} \log _{10}\left(\frac{I_{F 125}(\bar{v})}{I_{0}(\bar{v})}\right)
$$

where L is the absorption path length in the Herriott cell. Since nitrogen doesn't absorb at the wavelength of the ECQCL, the nitrogen reference scan provides an intensity background of the laser. Additionally, any absorption occurring in sections of the optical path other than the Herriott cell (e.g., due to water in the air between cell and detector) will be factored out.

\section{Results}

Figure 9 shows an experimental Freon-125 absorption scan taken using the arrangement and technique discussed in the previous section. The ECQCL was in fast scan mode and the Herriott cell path length was measured to be $1305 \mathrm{~cm}$. The solid line shows the experimentally measured absorption spectrum, and the dashed line shows a reference absorption spectrum ${ }^{(3)}$ obtained from the Northwest Infrared spectral library (Sharpe et al. 2004), which has been scaled for a 10-ppm concentration to correspond to the experimental gas mixture. We stress that there were no other adjustable parameters, and no other scaling actions performed. As can be seen from the figure, there is excellent agreement between the spectrum measured with the ECQCL and Herriott cell and the reference spectrum, both in spectral position and magnitude. The small offset between the spectra in the $1160-1180 \mathrm{~cm}^{-1}$ region is most likely due to a drift in the detector response during one of the scans.

(3) The "reference spectrum" of Freon-125 discussed here is different from the "reference scan" taken using N2 discussed in the Experimental Arrangement section. 


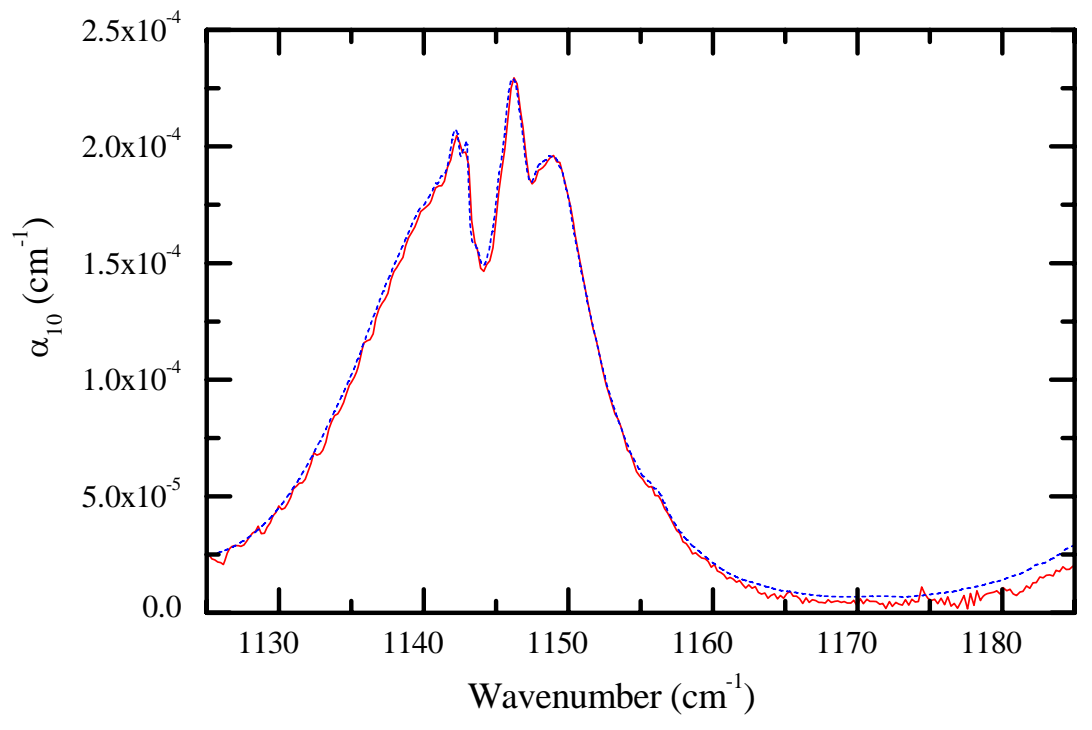

Figure 9. Measured Absorption Spectrum of 10 ppm Freon-125 in Fast Scan Mode. The solid red line is the absorption spectrum measured using the ECQCL and Herriott cell configured for a path length of $1305 \mathrm{~cm}$. The dashed blue line is a reference absorption spectrum obtained from the NWIR spectral library.

To increase the sensitivity of the Herriott cell sensor, the path length was increased by changing the mirror separation. Figure 10 shows the results of this increase. The solid line is the absorption spectrum of Freon-125 obtained in fast scan mode with the longer path length. The dashed line again shows the reference absorption spectrum. The increase in SNR is apparent over the results shown in Figure 9. An additional sharp feature can be seen in the absorption spectrum near $1175 \mathrm{~cm}^{-1}$. This feature is due to changing water vapor concentration in the room during the measurement, the absorption process occurring not in the Herriott cell, but in the portions of the infrared beam located outside the Herriott cell. This will be discussed further below.

Figure 11 shows the absorption spectrum measured using the high-resolution scan mode. The Herriott cell path length for this scan was determined to be $4702 \pm 1 \mathrm{~cm} .{ }^{(4)}$ Figure 12 shows the actual individual scans of Freon-125 (lower curve) and nitrogen (upper curve) before application of Eq. (1) to produce the absorption spectrum shown in Figure 11. The solid line is the transmitted signal with the Herriott cell containing Freon-125, and the dashed line is the transmitted signal with the cell containing pure nitrogen. In addition to the broad absorption feature due to Freon-125, two narrow absorption features are also present. These features were determined to result from absorption by water vapor in the beam path located outside the Herriott cell. In the case shown in Figure 11, the cancellation was nearly perfect, while the results shown in Figure 10 show an imperfect cancellation due to a changing water vapor concentration during the scans. However, even for the case of imperfect cancellation, the broad spectral feature from Freon-125 is easily distinguished from the narrow features.

(4) To determine the actual path length, a least squares fit of the experimental spectrum to the reference spectrum was performed with path length as a fit parameter. 


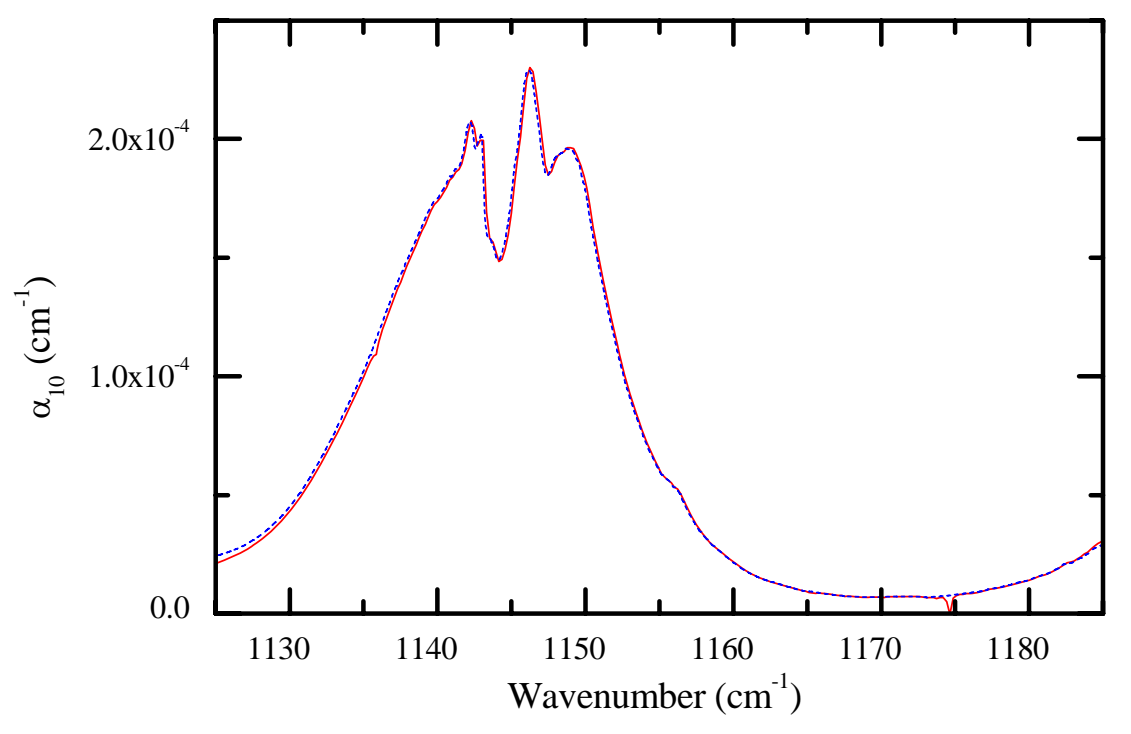

Figure 10. Fast Scan Absorption Spectrum of 10 ppm Freon-125. The solid red line is the absorption spectrum measured using the ECQCL and Herriott cell configured for a path length of $47 \mathrm{~m}$. The dashed blue line is a reference absorption spectrum obtained from the NWIR spectral library.

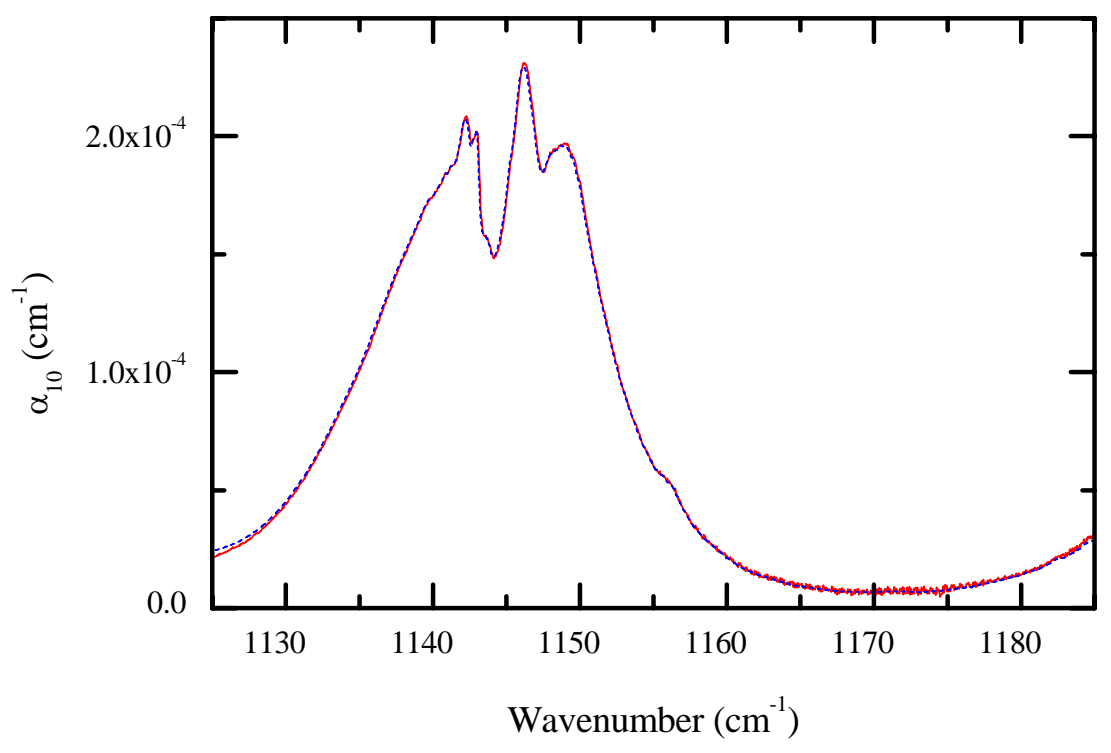

Figure 11. High Resolution Absorption Spectrum of 10 ppm Freon-125. The solid red line is the absorption spectrum measured using the ECQCL and Herriott cell configured for a path length of $47 \mathrm{~m}$. The dashed blue line is a reference absorption spectrum obtained from the NWIR spectral library. 


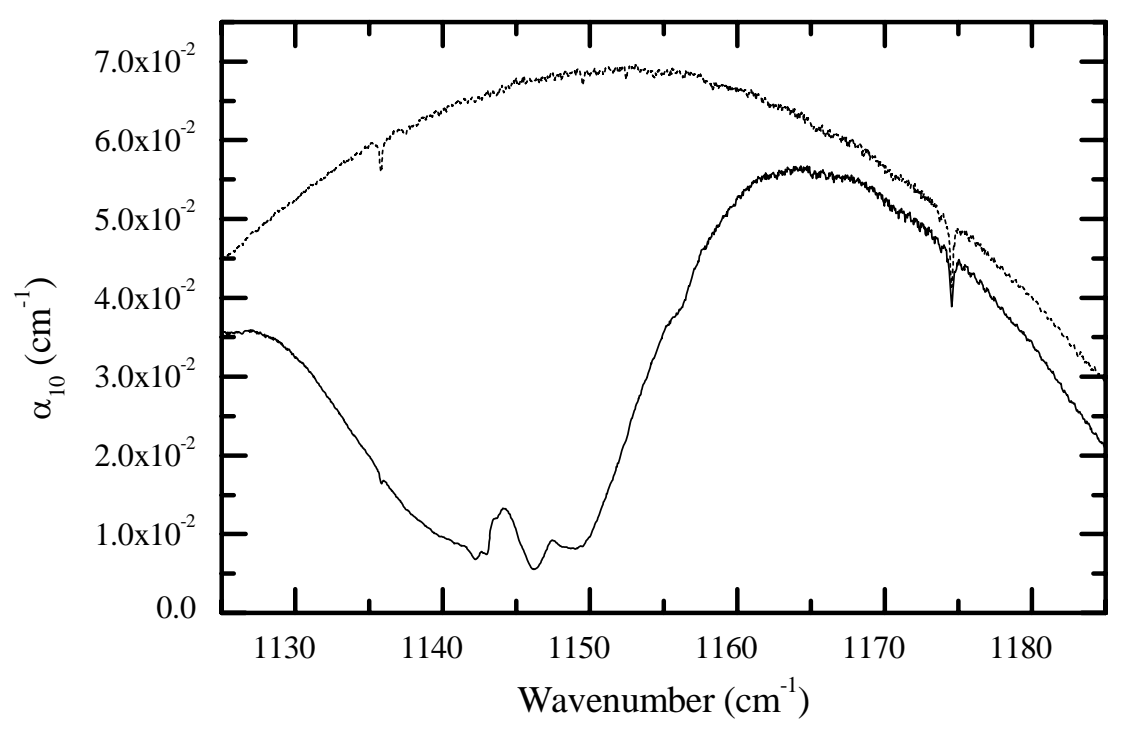

Figure 12. Individual Scans used to Calculate Absorption Spectrum. The solid line is a scan with 10 ppm F125 in the Herriott cell, the dashed line is with nitrogen in the cell. Two narrow water absorption lines are visible in the spectrum, due to absorption over the laser path length located outside the Herriott cell. The narrow lines are easily distinguished from the broad Freon feature.

To characterize the effects of potential interferents, the Herriott cell was filled with room air and an absorption spectrum was measured, shown in Figure 13(a). Figure 13(b) shows the absorption spectrum for water vapor, obtained from the NWIR spectral library. The peak positions and line shapes allow a positive identification of the absorption as due to water vapor in the air. A least-squares fit of the experimental absorption spectrum to the reference spectrum with water vapor concentration as the fit parameter yielded a concentration of $1.6 \%$. Using a saturation vapor pressure for water of 23.76 Torr at $25^{\circ} \mathrm{C}$, we calculated a relative humidity of $50 \%$, which is consistent with typical humidity levels in the laboratory. 


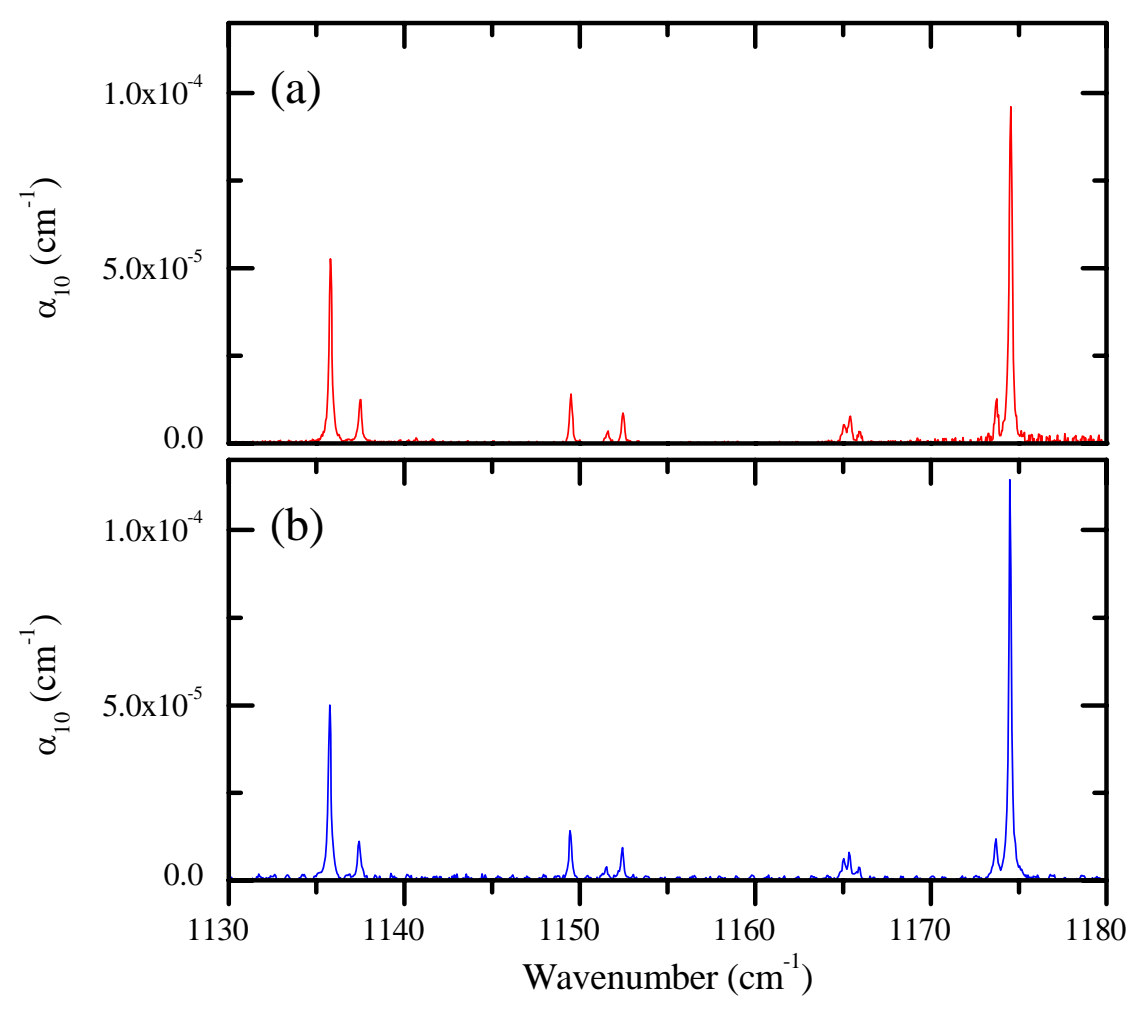

Figure 13. Absorption Spectrum of Room Air in Herriott Cell. (a) Experimental spectrum.

(b) Reference spectrum for water vapor from IR spectral library. A least-squares fit of experimental spectrum to the reference spectrum using water concentration as a parameter gives a water vapor concentration of $1.6 \%$ in the air.

\section{Summary and Discussion}

We have demonstrated that a simulant (Freon-125) approximating the spectral behavior of certain chemical agents can be detected using a spectrometer consisting of an external cavity quantum cascade laser and a multi-pass Herriott cell. The resulting spectra obtained were found to match spectra obtained from the Northwest Infrared Spectral Library very closely. The noise equivalent absorption sensitivity of the measurements was found to be in the range of $0.5 \times 10^{-7}$ to $1 \times 10^{-6} \mathrm{~cm}^{-1}$, corresponding to a relative concentration of 25 to 50 parts-per-billion by volume of Freon-125. The spectrum of a typical interferent, water, was also taken. The narrow features of such an interferent are easily distinguishable from the broad features of a target such as Freon-125.

It should be noted that the fulfillment of the goals of this task differs from the outline given in the lifecycle plan (LCP) in the following ways and for the following reasons. First, it was stated in the LCP that Freon-114B2 was to be used as the principal simulant (among others). This was our initial idea, attractive because of the presence of strong features in the 9.79-micron region, corresponding most closely to wavelengths of certain chemical agents. A QCL was also specifically obtained to operate in this region, although it did not arrive until Q2FY07, leaving little time to optimize ECQCL laser operation using this device. On the other hand, considerable investment had already been made in tunable 
laser development at 8.5 microns in the Laser Development sub-task. In order to carry out the CBW task exactly as written, it would have been necessary to re-engineer the ECQCL to operate with the new QCL, and purchase a separate supply of Freon-114B2. While neither presented any particular technical challenge, they would have required extra time and expenditure. It was recognized that given equal future availability of QCLs at either wavelength, the same demonstration could be performed at 8.5 microns using Freon-125, of which we already had plenty, without loss of generality or impact. This allowed us to complete the task on time and within budget. A second notable difference to the statements in the LCP is that of the interferents. The LCP mentions ozone $\left(\mathrm{O}_{3}\right)$. This was just one possible interferent, others including water. Due to time constraints and difficulty in obtaining and using $\mathrm{O}_{3}$ in the laboratory, it was opted to just observe the effects due to water. Again, as the features of both these simple compounds are narrow, the effects of $\mathrm{O}_{3}$ and other similar interferents on the spectra can be estimated just by observing those of water.

\section{References}

Littman MG and HJ Metcalf. 1978. "Spectrally narrow pulsed dye laser without beam expander (ET).” Applied Optics 17:2224.

Phillips MC, TL Myers and MS Taubman. 2005. FY 2005 Laser Development Final Report. PNNL15582. Pacific Northwest National Laboratory, Richland, Washington.

Phillips MC, TL Myers, MD Wojcik and BD Cannon. 2007. "External Cavity Quantum Cascade Laser for Quartz Tuning Fork Photoacoustic Spectroscopy of Broad Absorption Features.” Optics Letters. Doc. ID 78140, in press.

Sharpe SW, TJ Johnson, RL Sams, PM Chu, GC Rhoderick and PA Johnson. 2004. "Gas-Phase Databases for Quantitative Infrared Spectroscopy.” Applied Spectroscopy 58(12):1452-1461.

Taubman MS, BD Cannon, TL Myers, CA Bonebrake, PM Aker and JF Schultz. 2003a. Long Wave Infrared Cavity Enhanced Sensors. PNNL-14484. Pacific Northwest National Laboratory, Richland, Washington.

Taubman MS, TL Myers, BD Cannon, RM Williams and JF Schultz. 2003b. Ultra-Trace Chemical Sensing with Long-Wave Infrared Cavity-Enhanced Spectroscopic Sensors. PNNL-14201. Pacific Northwest National Laboratory, Richland, Washington.

Taubman MS, DC Scott, BD Cannon, TL Myers, CA Bonebrake, PM Aker, MD Wojcik, JT Munley, VT Nguyen and JF Schultz. 2004. Long Wave Infrared Cavity Enhanced Sensors. PNNL-15103. Pacific Northwest National Laboratory, Richland, Washington.

Taubman MS, DC Scott, BD Cannon, TL Myers, JT Munley, VT Nguyen and JF Schultz. 2005. Long Wave Infrared Cavity Enhanced Sensors. PNNL-15576. Pacific Northwest National Laboratory, Richland, Washington. 